\title{
New Socio-technical Devices to Improve Coordination in Healthcare in France
}

Therese Depeyrot-Ficatier ${ }^{1 *}$, Christian Bourret ${ }^{2}$ and Claudie Meyer $^{3}$

${ }^{1}$ Department of New Digital Value Consulting, DICEN IDF, France

${ }^{2}$ Department of Information and Communication Sciences, Deputy Director Research Team, France

${ }^{3}$ Department of Thematic Territory, Interactions, Mediations, France

\begin{abstract}
In the specific context of the Healthcare coordination between medical, socio medical and social organizations of the ambulatory field in France, we propose to examine some tracks with the perspective offered by new ways of sharing information. First we explain our researchers' position and the methodology used.

Then after reviewing different models of coordination entities, we intend to clarify the role played by such organizations acting as interface between hospitals and ambulatory services.

We will then address the issue of Information and Communication Technologies (ICT) that contribute to support and improve the healthcare coordination.

Through the evolution observed with the convergence between organizational models of coordination and technological devices, we will try to highlight the way of producing and sharing information on healthcare platforms for care coordination.

As an illustration of the value created by the new socio-technical devices, we will have a glimpse at the current innovative trend in healthcare coordination in France.
\end{abstract}

Keywords: Data sharing; Healthcare system; Care coordination; Interface organization; Information and Communication Technologies (ICT)

\section{Introduction}

The French Healthcare System is both characterized by divisions, especially between primary care and hospitalization sector and its essentially curative approach. For H. Isaac, Information and Communication Technologies (ICT) may improve its efficiency (traceability of patients' pathways) and do evolve it from a curative to a more preventive system [1].

The current prevalence of multi-chronic diseases has brought important changes: The stakeholders are more numerous; more complex factors such as the old age or the social elements have also to be taken into account; the duration of the patients stays in hospital are reduced; the healthcare system requires more and more coordination for professionals inside and between the structures, etc.

Thus, to establish fluidity in the collaboration between the healthcare providers, the structures and the patients has become essential. The coordination in healthcare assumes new shapes: It must connect not only medical professionals, but also all kind of healthcare providers and social workers; it has to be practiced for home healthcare at local levels in order to avoid the patients' hospitalizations; a territorial organization is needed for providing comprehensive and coordinated healthcare.

Coordination has become a key point. The coordination in healthcare is based on the exchange of information between the stakeholders: Those exchanges were till now mainly centered on the medical patient's information of the Electronic Healthcare Record (EHR); more and more information is now produced automatically through apps and connected devices by the patients themselves; "technology begins to integrate with the physical and biological, becoming more deeply intertwined in our lives [2]."

Taking care of the patients consists now in organizing their healthcare pathways around a personalized plan; it is necessary to “develop and update an individualized care plan $(\ldots)^{1}$ it should reflect current and long-term needs and goals for care; specify the types and frequency of all planned health $(\ldots)^{2}$, medications, home care services and supports, and other services; identify who is responsible for providing each service; and flag any critical issues that trigger a need to revise the plan."

We will deal mainly with the coordination structures acting as interfaces organizations between hospitals and the ambulatory services and bring out the specific issues in connection with the Information and Communication Technologies (ICT): The transition from the medical EHR to more expansive, frequent and updated information sharing, the new ways of sharing data between professionals within territorial cooperation; the transformation of practices and the development of interface organizations combined to digital healthcare platforms.

\section{Researchers' Position and Methodology}

We position in an Information and Communication Interdisciplinary Approach according to Bernard [3] with the convergence of the four aspects of link (interactions), meaning, knowledge and action. We act in a research action perspective to build new knowledge for action. So this paper associates a consultant in Healthcare Management especially with the key role of ICT (Information and Technology) and two University Researchers acting in Master specialized both in Social Protection especially in Healthcare and in Services Innovation.

*Corresponding author: Therese Depeyrot-Ficatier, Department of New Digital Value Consulting, DICEN IDF, France, Tel: 016095 7565; E-mail: t.depeyrot@ndv-consulting.com

Received August 16, 2017; Accepted August 24, 2017; Published August 28, 2017

Citation: Depeyrot-Ficatier T, Bourret C, Meyer C (2017) New Socio-technical Devices to Improve Coordination in Healthcare in France. J Health Med Informat 8 : 281. doi: $10.4172 / 2157-7420.1000281$

Copyright: (ㄷ 2017 Depeyrot-Ficatier T, et al. This is an open-access article distributed under the terms of the Creative Commons Attribution License, which permits unrestricted use, distribution, and reproduction in any medium, provided the original author and source are credited. 
We point out the importance of the notion of situation, particularly in the case of coordination's situation with information and communication challenges.

We insist both on the importance of communication and coordination in this approach and on the idea of organizing [4-24] to link both communication Sciences (and also information) and Management Sciences. Cooren and Robichaud [14] have in particular insisted of the Constitutive Role of Communication in Organizations with the importance of language at work and in the organizations. In France, this point has been especially developed by Borzeix and Fraenkel [9]. Cooren and Robichaud [14] insist also not only in the importance of human actors' role and interactions in the development of organizations but also on that of socio-technical devices in the way proposed by Akrich et al. [1] in an Actor Network Perspective. We point also the constitutive role of platforms [17] both to develop interactions and to propose new services in a coproduction and collaborative ways associating all the actors including the patients, integrating their emotions and feelings.

We actually consider organizations as ecosystems. So we propose to work in an ICOE (Information and Communication Organizing Ecosystems) perspective.

Our methodology tries to link observations in concrete situations and theoretical perspective defined above. Our observations in practice cases situations of activity in the French Healthcare System are sustained by interviews and gatherings of documents about activities (paper or Websites).

\section{Importance of Coordination and Role of Interface Organizations}

The development of the Coordination in Healthcare is an important challenge both to improve the quality of care (traceability in a process management perspective linked to Quality approaches) and to master the costs of care in all the developed countries and especially in France $[6,10]$.

- Different models of interface organizations between hospitals and ambulatory services can be analyzed through the evolution of the French healthcare system; they formed to respond to the lack of coordination and to cope with the needs of the patients with the reduction of the hospital stay duration, the incidence and prevalence of chronic diseases, the growth of the aging population and the multi morbidity among the elderly patients.
Care coordination has been defined as "the deliberate integration of patient care activities between two or more participants involved in a patient's care to facilitate the appropriate delivery of health care services.

$(\ldots)^{3}$ Care among multiple providers must be coordinated to avoid wasteful duplication of diagnostic testing, perilous polypharmacy, and confusion about conflicting care plans."

"Care coordination in the primary care practice involves deliberately organizing patient care activities and sharing information among all of the participants concerned with a patient's care to achieve safer and more effective care [4]."

The Figure 1 points out the different components of the function of care coordination.

- In France since about thirty years, the Healthcare Networks have enabled to organize a better ambulatory care delivery for some diseases (after the first Healthcare Networks for AIDS at the beginning of the years 1980, others set up for diabetes, cancer, and ante postnatal care, etc.).

The Healthcare Network is the first model of coordination in the French ambulatory healthcare system. Its main goal is to organize the coordination between the hospital and all the stakeholders involved in the patients' home nursing and caring, playing the role of interface organization.

Its distinctive feature is the creation of connections between the different healthcare providers affected by the patient centered home care but not working in the same place. In this objective, they define common care protocols and organize training sessions, meetings.

After the innovative first period, through their financing, the healthcare policy orientated their development with the aim of dealing with several pathologies or situations (cancer and palliative care...) and covering more extended territorial areas [5].

- The multidisciplinary medical homes (Maisons de Santé Pluri professionnelles ou pluri disciplinaires MSP) represent another model of coordination in the ambulatory field with care protocols shared by healthcare providers gathered together in the same place.

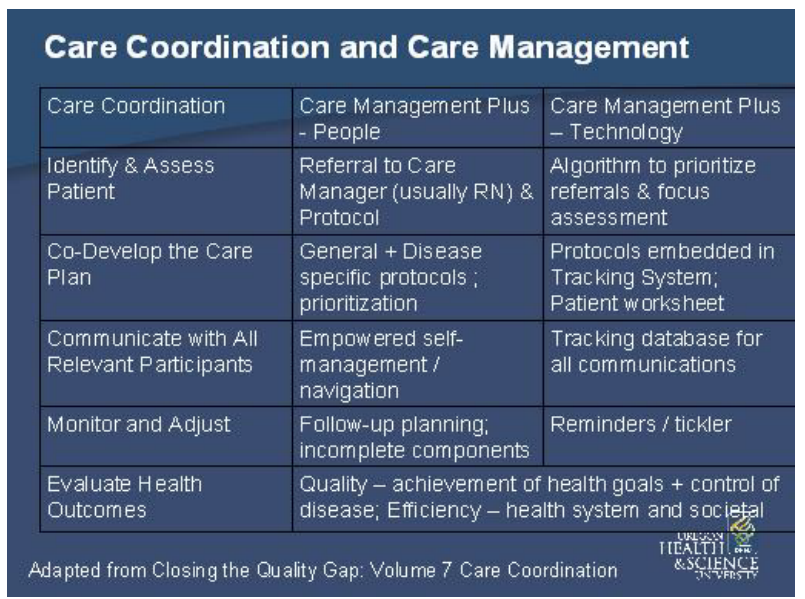

Figure 1: Presentation from the Agency for Health Research and Quality (AHRQ) 2007 annual conference. 
Emphasizing «the components of coordination $(\ldots)^{1-4}$, the shared protocols " combine medical, caregiver, and medical social expertise to better manage a situation concerning one or more acute or chronic illnesses. It responds to a multidisciplinary issue identified by a team, within a healthcare facility or in an area. $(\ldots)^{5}$ This group must include members belonging to all the professions concerned: $(\ldots)^{6}$ primary care team, medical social professionals and second line [6] specialists $(\ldots)^{7} »$.

Some MSP now implement telehealth projects, such services strengthening links with hospitals.

- The model Asalée (Action de santé libérale en équipe) is a special case of coordination between healthcare providers working in the same place. With the raise of aged patients and the increase of chronic diseases, using skill mix in Primary Healthcare (PHC) and providing accurate protocols for activity transfers to different healthcare providers helps to save the medical time in a context of scarce medical resources [7].

This model Asalée came up in 2004 in a primary care organization with a cooperation protocol between general practitioners and nurses for improving care for patients with chronic conditions. First orientated towards the diabetes monitoring, the protocol has been extended to screening for memory troubles and Chronic Obstructive Pulmonary Disease (COPD) and to the follow-up care for cardiovascular disease [8].

- The hospital at home structures (Hospitalization A Domicile HAD) play a part in avoiding in-patient hospital care; they have to involve multidisciplinary teams to provide care at home. Furthermore, they also act as interface organizations in the ambulatory field: they have an important role in coordination particularly at some critical periods of the patient's healthcare pathway: admission, discharge and re-admission in hospitals.

In this context, the hospital at home structures have to establish connections with the healthcare providers and social workers involved: at the upstream phase, in the hospital where the patient comes from; at the downstream one, with the structure where he is hospitalized. When the patient recovers his health, the link is to create with home care services (Services de Soins Infirmiers A Domicile SSIAD) for the next episode in his healthcare pathway [9].

- More recently in France, homes for independent living by Alzheimer's patients (Maisons pour l'Autonomie et l'Intégration des Malades d'Alzheimer MAIA) have been created for specific issues for elderly patients. Based on the Case Management principles and practices, they are now named Action method for the integration of the cure and care services for the autonomy field (Méthode d'Action pour l'Intégration des services d'aide et de soins dans le champ de l'Autonomie MAIA).

They play a full part in the coordination of all the healthcare providers for patients with complex needs; therefore they get into position of an interface organization between the hospital and the ambulatory field. With an orientation towards person-centered care, after the patients' orientation and inclusion, their main function is the drawing up of the individualized healthcare plan (Plan Personnalisé de Santé PPS).

- The French Healthcare and Social Affairs Ministry decided to develop new organizational models for the healthcare pathway for elderly patients with risk of loss of autonomy (Parcours de santé des personnes Agées En Risque de Perte d'Autonomie PAERPA). Nine regional pilots have been launched in 2014 in territories with the aim of improving the healthcare and living conditions of older people aged over 75 years old and the coordination for frail elderly people, especially between the medical sector and the social care.

Care coordination is ensured by a dedicated team (Coordination Territoriale d'Appui CTA): its main functions are information and orientation, support for tracking and screening risk of loss of autonomy, help for the creation of the local clinical coordination (Coordination Clinique de Proximité CCP), support for drawing up the PPS, help for access to social rights and assistance and for strengthening connections with hospital for admissions, re-admissions and discharges.

With the emergence of new territorial organizations for the coordination between hospitals and ambulatory field, the risk is that their superposition may occur without a sufficient redefinition of their roles in order to enable structuring the prefiguration of the future healthcare system.

- The Information and Communication Technologies (ICT) contribute to a great extent to improve the healthcare coordination.

The first step has been the implementation of Electronic Health Records (EHR), helping to share information about the patients among doctors and more widely among healthcare providers.

In 2004, the French government started the project of personal medical records (Dossier Médical Personnel DMP) in order to centralize all the medical documentation: discharge letters, results, consultation notes and patient summaries... Since 2016, the DMP is named the shared medical record (Dossier Médical Partagé) and its management has been given to the Health Insurance, which develops an important communication campaign to promote it, and it is experimented in some departments.

While waiting for the general implementation of this EHR, the use and sharing of other electronic healthcare records expanded.

The Healthcare Networks had a part in this evolution, mainly with medical specialty records (for instance, diabetes...); in this context, the electronic cancer record (Dossier Communicant de Cancérologie DCC) began to be structured, as a specific tool for the multi-disciplinary coordination meetings (réunions de concertation pluridisciplinaire RCP).

As for the use of EHR for the coordination, according to practices, EMRs (Electronic Medical Records) "are less able to support coordination between clinicians and settings, in part due to their design and a lack of standardization of key data elements required for information exchange; $(\ldots)^{8}$ managing information overflow from EMRs is a challenge for clinicians; $(\ldots)^{9}$ clinicians believe current EMRs cannot adequately capture the medical decision-making process and future care plans to support coordination.»

With the growing need for delegation and skill mix in healthcare teams, the development of cooperation between the healthcare professionals, sharing information has become a critical concern. In the model Asalée that is now extended in France, the nurses share the patients' EHR with the general practitioners for tracking the patients with risk factors and organizing the screening for chronic diseases, recording their consultation reports, the evaluation results and the warnings. 
With the Information System (IS), they have at their disposal monthly indicators dashboards for measuring the activity, the clinical characteristics and pathological distribution, and data elements for the patients' monitoring.

For efficient care coordination, the information about the patient has to be recent and synthetic; for answering their needs, the healthcare providers must be able to access to the right information at the right moment and to share the essential for taking care and follow up the patients.

Some tools have been defined for improving the way of sharing the right information with the other healthcare providers in the elderly patients' pathway PAERPA, besides the EHR (DMP): the healthcare personalized plan (Plan Personnalisé de Santé PPS), the medical synthesis (Volet de Synthèse Médicale VMS), and specific records helping for an essential link in case of an emergency situation (Dossier de Liaison Urgences DLU).

"A personal health record is an electronic source of a patient's health information that can be used by the patient or a proxy. When personal health records are linked with patients' electronic health records, they have the potential to transform the care of chronic conditions, particularly because they can help engage people more fully in aspects of their care."

With chronicity, patients are more involved not only for getting access to their medical records, but also for recording themselves their personal healthcare data.

Some digital solutions are available for recording healthcare indicators such as high blood pressure... and for handling the storage and sharing of healthcare personal information in complete digital records.

"As more patient data gets liberated from isolated systems and added to networks, comparable knowledge and value can be generated for healthcare [10]."

"...Disruptive innovation $(\ldots)^{10}$ inverts the current approach to medical records $(\ldots)^{11}$. Personal Health Records combined with social networking $(\ldots)^{12}$ may lead to a powerful new generation of health applications, where people share parts of their electronic health records with other consumers and "crowd source" the collective wisdom of other patients and professionals. (... $)^{13}$ Conceptual model of a second generation (Table 1) of personal health records, which not only allows patients to access their electronic health record, but to share parts of it with other people, building communities around certain health topics and issues [11].»

\section{In a New Territorial Approach of Coordination}

- Through the evolution observed with the convergence between organizational models of care coordination and the ICT structures, we to highlight the way of producing and sharing important volumes of data on healthcare platforms for the coordination.

As the French Healthcare Ministry makes reforms for implementing the transformation of the healthcare system through experimental models, we will first consider the evolution from the program for digital healthcare territories (Territoire de Soins Numérique TSN) to the support territorial platforms (Plateformes Territoriales d'Appui PTA) and more recently the digital services for coordination support (Services Numériques d'Appui à la Coordination SNAC).
- TSN is a national health information technology-based program "aiming at improving healthcare coordination and access to information for healthcare providers, patients and the population, and at improving healthcare professionals work organization $(\ldots)^{14}$. In 2014, a governmental grant called TSN (Territoire de Soins Numérique/Digital health territories), selected five Healthcare Information technology development projects across five pilot areas containing between 200,000 and 500,000 inhabitants each in five different French regions [12] $(\ldots)^{15 ”}$

The five regional experimental projects have answered the common goal of improving the healthcare pathways organization through the implementation, in a territorial frame, of a configuration for care coordination support.

- The TSN program enabled to set up a prefiguration of the PTA platforms that were then defined in 2016 with specific functions in support to healthcare providers for the coordination in the complex healthcare pathways [13]

Based on the extension and redefinition of existing organizations, the PTA platforms have to foster the care coordination of all the activities of the healthcare providers, especially during hospital admissions, hospital leaving, and during the critical period of readmissions.

"Transitions, such as from the hospital to a rehabilitation facility or from a rehab facility to home, are particularly vulnerable times for patients [14]."

As devices for care coordination support to the healthcare professionals in complex pathways, the PTA have to be structured mainly by the healthcare providers in the ambulatory field; they may have constituted a territorial community (Communauté Professionnelle Territoriale de Santé CPTS) for managing their common project in order to answer the healthcare needs and provide services.

The example of the Brittany ARS (Bretagne) points out the priority given to the information sharing in the development of the healthcare pathways. Currently, a project aims to implement a shared Information System for the healthcare professionals in coordination teams in order to enable them to exchange data on patients and collect evaluations for programming PPS. The ARS wants to display this connected devices to

\begin{tabular}{|c|c|}
\hline Type of E health services & E Health services functionalities \\
\hline \multirow{3}{*}{ Organisation of Healthcare } & Platform for service coordination \\
\hline & Digital coordination service \\
\hline & Call center for informal caregivers \\
\hline \multirow{4}{*}{ E-services for users/patients } & Diffusion service of general healthcare information \\
\hline & Medical information management services \\
\hline & Administrative information management services \\
\hline & Community of connected patients \\
\hline \multirow{4}{*}{ E-services for professionals } & General health information service \\
\hline & $\begin{array}{l}\text { Professionals patient medical information } \\
\text { management }\end{array}$ \\
\hline & Administrative information management services \\
\hline & Community of connected professionals \\
\hline \multirow{4}{*}{$\begin{array}{l}\text { Administrative and } \\
\text { monitoring services for } \\
\text { policy makers }\end{array}$} & Administrative monitoring \\
\hline & Epidemiological monitoring \\
\hline & eHealth innovation promotion \\
\hline & Interoperability management \\
\hline
\end{tabular}

Table 1: Presentation of digital/E-services and functionalities in each TSN project area. 
different types of structures and stakeholders. Several MAIA cover the entire region; in addition, PAERPA have been set up on three territories and the first two PTA have been created from eight Healthcare networks. Healthcare professionals themselves have launched another. Seven PTA are now in prefiguration.

- In 2017, the French Healthcare ministry launched a call for regional projects for the progressive implementation of digital services in support to both usual and complex care coordination (Services numériques d'appui à la coordination SNAC).

The projects integrates the existing organizations offering support functions to care coordination (Healthcare networks, MAIA, PAERPA, PTA) with another step in the extension of the previous programs. In the selected territory, the digital services have now to address the different types of populations and must be supported by healthcare professionals from public and private sectors in the healthcare, medico social and social fields. The Healthcare regional agencies (Agences Régionales de Santé ARS) will select their pilot territories for trialing the model before the regional generalization.

The digital services displayed on SNAC are basic and priority (Table 2) services, some are imperative for the management of the healthcare pathways and the coordination, as mentioned below:

While the regional calls for projects are launched, orientations are defined for SNAC: in the region Ile de France for instance, the extension of TSN has to enable the development of new organizations and uses: the digital services must improve the information sharing, the healthcare pathway organization through innovative collaborative practices between the healthcare providers. ICT should help to anticipate the acute care episodes in order to avoid hospitalizations, with uses of medical telemonitoring for connecting with the care providers and remote surveillance with warning reports.

\section{Results and Discussion}

The recent evolution in the French healthcare system is the confirmation of the role played by socio-technical devices and especially the platforms. The interface organizations acting between the hospitals and the ambulatory field (primary care) try to create intermediation that is enabled through ICT. They constitute innovative areas. The use of digital devices contributes efficiently to the development of innovative and opened organizations. A current innovative trend seems to emerge in healthcare coordination in France through the observation of the platforms as new healthcare socio-technical devices.

With main functions as intermediation, shared (mutualisés) services, resources and information sharing, the aims of the healthcare digital platforms define them as organizational objects rather than ICT tools, with a real value creation through information sharing and collaborative practices.

\section{Digital basis health care services}

Digital services for the healthcare pathway and care coordination management Tracking evaluation of the patient's condition

Professional network

Coordination records, PPS

Warnings, event notifications

Shared scheduling, medical appointments, hospital admissions

Tools for help in orientation and programming

Management of the resources in support the care coordination

Table 2: Digital basis health care services.
The healthcare platforms offer a strong interweaving between organization and technology and digital services are the support for the care coordination that is essential for coproduction and co innovation.

The use of technical devices takes part in the evolution of the professional practices; all those perspectives draw the outlines of "a creative destruction" of the healthcare system, with new forms of care organizations based on remote information exchanges [15].

Huge volumes of data, and more particularly the data flows, giving an image of the real world at a given instant and in its dynamic, it enables a more efficient achievement of the intermediation [16].

In the new digital culture, M. Doueihi insists on the new role and the importance of the platforms: they are essential none only for managing the date access and storage, but because they become, thanks to the users' activities, places of convergence between information, communication, knowledge and sociability [17].

The digital intermediation will partly belong to huge nonspecialized platforms as Google, Amazon... but it will leave space for specialized ones acting locally [18].

In the information flow and sharing is found the idea of transition from the information production at the individual level to the collective production that integrates the knowledge of all the stakeholders.

The digital transition now enables to rethink the model of healthcare system. Agility can be developed with the intermediation platforms supporting the management of networking communities that fosters the collective intelligence.

"The systemic changes brought by the digital revolution with ICT are considerable. For really taking advantage of the digital turn, there is not only to implement solutions based on technologies, but also to establish a real innovation culture. $(. . .)^{16}$ For a successful transition towards digital healthcare, one of the first tracks to foresee is the help and support for a strengthening a multi-disciplinary approach [19]. »

\section{Conclusion and New Insights}

The new socio-technical devices consist in a strong combination of organizational and technical intertwined components; they enable the increasing patients' involvement in the healthcare data producing and exchanging: software now commonly track the patients' transactions on websites and their feedbacks on social networks..

"Enabled by technology," $(\ldots)^{17}$ the interface [20] organizations are moving from $(\ldots)^{18}$ provider centric to $(\ldots)^{19}$ user centric." The patients are more and more involved for their healthcare through the use of ICT, making inquiries on digital healthcare platforms, producing, recording or sharing their own personal data, exchanging with other patients or professionals on healthcare social networks...The new ways of data sharing between healthcare professionals rest on the new tools: shared healthcare records, digital healthcare platforms, specialized call centers...

With the huge amount of data produced and exchanged through the new socio-technical healthcare devices, the security challenge has to be addressed; "Current limitations around security, integrity, and access control for personalized health data are creating critical bottlenecks for care delivery innovations. This, in turn, creates an inefficient digital health workflow with acute data silos between different providers $(\ldots)^{20}$ impeding care coordination. $(. . .)^{21}$ Potential application of blockchain technology provides a timely solution to mitigate some of these pressing needs. $(\ldots)^{22}$ It could help the healthcare industry resolve some of its pressing challenges, such as cyber security, data interoperability [21]." 
Current decisive issues may lead to a deep mutation for the French healthcare system.

The improvement in the organization of the patients' healthcare pathways brings a transition toward a real cooperation between all the stakeholders at the service of the patients, in a context constrained by limited resources, with the passage from merely data sharing to developing a collective intelligence in logic of services co-innovation.

The healthcare interface organizations constitute privileged spaces of innovation. "Creativity and innovation acquire a new centrality in the contemporary scene $(\ldots)^{22}$. The new digital platforms are essential components in this process, extending the possibilities of interaction and networked production [22-24]. Territories, on the other hand, gain informational, communicational, and technical density, constituting privileged spaces for social experimentation $(\ldots)^{22}$."

We are also in a transition time with all the importance and the ambivalence of the technology (Ellul); it can improve the system but can also create new constraints corresponding to a form of services "taylorization" with great problems of suffering at work.

The so-called French Healthcare Challenge is actually very compartmentalized and divided. It does not work as a system: oppositions, conflicts, any coherence, issues of traceability, etc.

ICT developed in a new territorial management approach may help this evolution from a curative to a predictive system (Isaac) with new role of patients (empowerment).

It is also the issue to evolve from coordination to cooperation and from participative to contributive actions, with new uses of data. We have also the specific issue of evaluation of these innovative devices and organizations.

The French Healthcare system is at a crossroads. It must change to survive betting on new socio-technical devices corresponding to e-health to first improve coordination and then build new form of co-operations in a proximity approach including patients (especially their needs, emotions and feelings) to tackle another challenge, that of overpass social and territorial inequalities in healthcare.

\section{Authorship}

Therese Depeyrot-Ficatier collected the observations about the projects and socio-technical devices, Christian Bourret [11,12] ret brought the main concepts for reflection and provided the insights, Claudie Meyer completed this work.

\section{Funding}

The researcher on this manuscript received no particular funds from any particular organization or research body.

\section{Conflict of Interest}

There are no conflicts of interest.

\section{Ethical Approval}

This article did not need any ethical approval as it did not deal with human participants or animals.

${ }^{1}$ Libert B (2012) Digital platforms are the key to improving your health in 2017 . Forbes January 4, 2017.

${ }^{2}$ Coordinating care for adults with complex care needs in the patient-centered medical home: challenges and solutions. January 2012, Agency for Healthcare Research and Quality (AHRQ) Publication No. 12-0010.

\section{${ }^{3}$ Bodenheimer [8]}

${ }^{4}$ https://www.ahrq.gov/professionals/prevention-chronic-care/improve/ coordination/index.html

${ }^{5} \mathrm{RN}$ : Registered nurse.

${ }^{6}$ Developing and implementing shared care protocols, French National Authority for Health, February 2015.

${ }^{7}$ In French, Caisse Nationale d'Assurance Maladie et des Travailleurs Salariés (CNAMTS)

${ }^{8}$ O'Malley AS, Grossman JM, Cohen GR, Kemper NM, Pham HH (2010) Are electronic medical records helpful for care coordination? Experiences of physician practices. Journal of General Internal Medicine 25: 177-185

${ }^{9}$ Bates DW, Bitton A (2010) The future of health information technology in the patient-centered medical home. Health Affairs 29: 614-621.

${ }^{10}$ Bringing the Power of Platforms to Health Care by Jonathan Bush and John Fox, Harvard Business Review, Technology, November 10, 2016

${ }^{11}$ Eysenbach G (2008) Medicine 2.0: social networking, collaboration, participation, apomediation, and openness. Journal of Medical Internet Research 10.

${ }^{12}$ Saillour-Glénisson F, Duhamel S, Fourneyron E, Huiart L, Joseph JP, et al. (2017) Protocole of a controlled before-after evaluation of a national health information technology-based program to improve healthcare coordination and access to information. BMC Health Services Research 17: 297.

${ }^{13}$ Source: https://doi.org/10.1186/s12913-017-2199-y

${ }^{14}$ Bates DW, Bitton A (2010) The future of health information technology in the patient-centered medical home. Health Affairs 29: 614-621.

${ }^{15}$ Cooren F, Fairhurst GT (2009) Dislocation and stabilization: how to scale up from interactions to organization, in putnam (Linda L.). In: Nicotera (Anne Maydam) editors,

${ }^{16}$ Enquête sur une révolution annoncée» Etienne Minvielle Le Libellio d' AEGIS Vol 11 n² 2 Été 2015 Dossier Santé Numérique pp: 13-29.

${ }^{17}$ Qu'est-ce que l'intermédiation algorithmique ? » Stéphane Grumbach, Inria 1024 Bulletin de la société informatique de France, Numéro 7, Novembre 2015, pp: 93-111.

${ }^{18} \mathrm{http}$ ://www.hbrfrance.fr/chroniques-experts/2016/05/10459-pourquoi-lenumerique-bouleverse-le-secteur-des-services/

${ }^{19}$ La santé à l'ère du numérique et les nouveaux rôles du patient Cathy Bazinet | Juin 1, 2017 | Santé numérique

${ }^{20}$ Libert B (2017) Digital platforms are the key to improving your health in 2017.

${ }^{21}$ Top 5 Reasons Why Every Healthcare Company Should Invest in Blockchain Reenita Das, Forbes, August 8, 2017

${ }^{22}$ Albagli S (2015) The "Creative Turn". Creative Capitalism, Multitudinous Creativity: Radicalities and Alterities, 175.

\section{References}

1. Akrich M, Callon M, Latour B (2006) Sociologie de la traduction. Textes fondateurs, Les Presses Mines de Paris 1: 304.

2. Albagli S (2015) The "Creative Turn". Creative Capitalism, Multitudinous Creativity: Radicalities and Alterities 1: 175.

3. Bates DW, Bitton A (2010) The future of health information technology in the patient-centered medical home. Health Aff 29: 614-621.

4. Bates DW (2010) Getting in step: electronic health records and their role in care coordination.

5. Bernard $F(2006)$ It is the discipline of the ouverture and the décloisonnement In: Bouzon A (eds.) dir, La communication organisationnelle en débat. L'Harmattan 25: 33-46.

6. Bloch MA (2015) Sur le chemin des parcours intégrés, Revue Réseaux, Santé \& Territoire, $n^{\circ} 641: 20-23$

7. Bloch MA, Hénaut $L$ (2014) Coordination et parcours. La dynamique du monde sanitaire, social et médico-social. Paris Dunod 1: 336.

8. Bodenheimer T MD (2008) Coordinating care-a perilous journey through the health care system. The New England Journal of Medicine pp: 1064-1071

9. Borzeix A, Fraenkel BC (2001) Langage et Travail. Communication, cognition action, Paris, CNRS Editions, Rééd 2005: 379

10. Bourret C (2016) Relever le défi des Inégalités Sociales et Territoriales en Santé (ISTS) par la rencontre des organisations d'interface et de la télésanté dans une. Approche d 1: 21-25. 
Citation: Depeyrot-Ficatier T, Bourret C, Meyer C (2017) New Socio-technical Devices to Improve Coordination in Healthcare in France. J Health Med Informat 8: 281. doi: 10.4172/2157-7420.1000281

11. Bourret C (2016) Nouveaux métiers d'intermédiation dans les organisations d'interface en santé, Ecosystème de santé: nouveaux modes de régulation de I'information, in Dufour-Coppolani D. and Hassanaly P. Information 1: 32-33.

12. Bourret C (2015) Les frontières organisationnelles comme espaces d'innovation dans le secteur de la santé, in Communication et débats publics: les réseaux publics au service de la démocratie. VREN 2013: 75-84.

13. Burton LC, Anderson GF, Kues IW (2004) Using electronic health records to help coordinate care. Milbank Quarterly 82: 457-481.

14. Cooren F, Robichaud D, (2011) Les approches constitutives, in Grosjean S. e Bonneville L. dir., La communication organisationnelle. Approches, processus et enjeux, Montréal. Chenelière Education 25: 140-175.

15. Cooren F, Fairhurst GT (2009) "Dislocation and Stabilization: How to Scale Up From Interactions to Organization". New York and London 1: 117-151.

16. http://nebula.wsimg.com/70e02a410579259c2e23e53616bca1fa?AccessKey $\mathrm{d}=3$ F9619340B04FB0BB6FF\&disposition=0\&alloworigin=1

17. Doueihi M (2011) Pour un humanisme numérique, Paris, Seuil-La Librairie du XXlème siècle, $\mathrm{p}: 178$
18. Dufour-Coppolani D, Hassanaly $P$ (2016) Ecosystème de santé: nouveaux modes de régulation de l'information, i2D-Information. Données et Documents 3: $28-69$.

19. Ellul J (1990) La technique ou l'enjeu du siècle, Paris, Economica, 1990, rééd. 2001: 423.

20. Eysenbach G (2008) Medicine 2.0: social networking, collaboration, participation, apomediation, and openness. J Med Internet Res 10: 22.

21. http://fr.slideshare.net/RenaissanceNumerique/lb-sante-preventiverenaissance-numerique-1

22. O'Malley AS, Grossman JM, Cohen GR, Kemper NM, Pham HH (2010) Are electronic medical records helpful for care coordination? Experiences of physician practices. J Gen Intern Med 25: 177-185.

23. Saillour-Glénisson F, Duhamel S, Fourneyron E, Huiart L, Joseph JP, et al. (2017) Protocole of a controlled before-after evaluation of a national health information technology-based program to improve healthcare coordination and access to information. BMC Health Serv Res 17: 297.

24. Weick KE (1989) Organized improvisation: 20 years of organizing Communication Studies 40: 241-248. 